\title{
Malate Dehydrogenase
}

National Cancer Institute

\section{Source}

National Cancer Institute. Malate Dehydrogenase. NCI Thesaurus. Code C116405.

A family of enzymes whose members reversibly catalyze the oxidation of malate to oxaloacetate using the reduction of $\mathrm{NAD}+$ to $\mathrm{NADH}$ and are involved in multiple metabolic pathways. There are 2 proteins in this family, malate dehydrogenase, cytoplasmic and malate dehydrogenase, mitochondrial. 